\title{
Colavita dominance effect revisited: the effect of semantic congruity
}

\author{
Alexandra Stubblefield • Lauryn A. Jacobs • \\ Yongju Kim • Paula Goolkasian
}

Published online: 14 August 2013

(C) Psychonomic Society, Inc. 2013

\begin{abstract}
To investigate the effect of semantic congruity on audiovisual target responses, participants detected a semantic concept that was embedded in a series of rapidly presented stimuli. The target concept appeared as a picture, an environmental sound, or both; and in bimodal trials, the audiovisual events were either consistent or inconsistent in their representation of a semantic concept. The results showed faster detection latencies to bimodal than to unimodal targets and a higher rate of missed targets when visual distractors were presented together with auditory targets, in comparison to auditory targets presented alone. The findings of Experiment 2 showed a cross-modal asymmetry, such that visual distractors were found to interfere with the accuracy of auditory target detection, but auditory distractors had no effect on either the speed or the accuracy of visual target detection. The biasedcompetition theory of attention (Desimone \& Duncan Annual Review of Neuroscience 18: 1995; Duncan, Humphreys, \& Ward Current Opinion in Neurobiology 7 : 255-261 1997) was used to explain the findings because, when the saliency of the visual stimuli was reduced by the addition of a noise filter in Experiment 4, visual interference on auditory target detection was diminished. Additionally, the results showed faster and more accurate target detection when semantic concepts were represented in a visual rather than an auditory format.
\end{abstract}

A. Stubblefield $\cdot$ L. A. Jacobs $\cdot$ P. Goolkasian

University of North Carolina, Charlotte, NC, USA

Y. Kim

Korea Military Academy, Seoul, South Korea

P. Goolkasian $(\bowtie)$

Department of Psychology, UNC Charlotte, 9201 University City

Blvd, Charlotte, NC 28223, USA

e-mail: pagoolka@uncc.edu
Keywords Visual Dominance effect · Cross-modal effects . Semantic congruency $\cdot$ Audiovisual

Processing real-world events involves multiple sources of information, and there is no doubt that such rich sensory stimulation enhances perception of those events. Interestingly, however, research with multisensory stimuli does not always result in performance advantages as compared to unimodal stimulus events (Colavita, 1974: Koppen, Alsius, \& Spence, 2008; Lee \& Chan, 2008). One notable example is the Colavita visual dominance effect (Colavita, 1974), which shows that when simple audiovisual stimuli are presented and targets appear in both modalities unexpectedly, visual targets are detected more often than auditory targets. The visual dominance effect occurs even though detection accuracy with unimodal testing conditions is comparable across modalities. The finding has been robust in spite of variation in stimulus intensity (Colavita, 1974; Lee \& Chan, 2008) and directed attention to each of the modalities (Koppen et al., 2008; Sinnett, Spence, \& SotoFaraco, 2007). However, there is considerable variation in the responses to bimodal and unimodal events when the task changes from the speeded modality discrimination task used in the original study by Colavita to a speeded detection task (Sinnett, Soto-Faraco, \& Spence, 2008) and when the task requires the use of one or three response buttons (Molholm, Ritter, Javitt, \& Foxe, 2004; Sinnett et al., 2008). Speeded detection responses with one response key, in particular, show faster facilitated responses to bimodal than to unimodal events, an effect that is referred to as the redundant-target effect.

It is indeed intriguing that the detection responses to bimodal events can change so dramatically from visual dominance, which is essentially an interference effect, to a facilitation effect with such modest changes in task parameters. The present study builds on recent efforts (Ngo, Sinnett, SotoFaraco, \& Spence, 2010; Sinnett et al., 2008) by testing whether the phenomenon of visual dominance over audition 
is also demonstrated when participants are asked to detect a target concept rather than a specific stimulus item within a modality.

Ngo et al. (2010) have recently shown that visual dominance over audition can occur when participants are asked to detect stimulus events that are defined by an abstract rule. Audiovisual streams of objects and environmental sounds were presented, and participants were instructed to detect an immediate repetition of a stimulus item by pressing one of three response keys in order to indicate whether the repeated item was an audio, visual, or bimodal event. Although overall more visual than auditory or bimodal targets were missed, when the bimodal errors were analyzed separately, the results showed that bimodal targets generated three times as many "visual" as "auditory" target responses. This finding was the first to demonstrate the visual dominance effect with a task that required processing beyond low-level sensory detection.

In other relevant work, Koppen, Alsius, and Spence (2008) tested whether semantic congruency modulated the Colavita dominance effect. They conducted a series of studies with pictures and sounds and manipulated whether the stimuli presented simultaneously were from the same or from different animals, such as the sound of a cat "meowing" paired with the picture of a cat (same) or a dog (different). The results showed that detection performance was faster and more accurate with congruent than with incongruent pairs, but that semantic congruency had no influence on the visual dominance effect. In the bimodal conditions, more visual than auditory targets were identified.

The finding that the presence of a visual target interferes with the detection of an auditory target, irrespective of semantic congruency, is somewhat at variance with others that have shown the influence of semantic congruency with bimodal targets (Chen \& Spence, 2010; Molholm et al., 2004; Suied, Bonneel, \& Viaud-Delmon, 2009). When researchers (Molholm et al., 2004; Suied et al., 2009) studied the combined influences of an animal picture and sound on object identification, they found faster and more accurate responses when bimodal events matched than when the targets appeared in only one sense modality. Similarly, Chen and Spence (2010) found that congruent sounds improved picture identification, whereas incongruent sounds interfered. Additionally, some brain research (Molhom et al., 2004; Murray \& Spierer, 2009) has demonstrated that auditory processing can occur within the same time frame as visual processing and that multisensory effects are present during early stages of the object recognition process.

Recent work by Zmigrod, Spapé, and Hommel (2009) suggested that auditory and visual features can be integrated and bound with each other and with their associated responses. Their data showed that feature integration operates across perceptual domains and that the mechanism that underlies multimodal sensory integration is an event file.
Originally proposed by Hommel (2004, 2005), an event file is a network of bindings that link salient feature codes of a perceptual event. Repeated encounters with a stimulus or with one or more of the bound features activates an event file, which either produces retrieval of the event file to facilitate performance, if the same stimulus event is experienced, or may interfere with the creation of new event files, for events that share some but not all of the same features. Event files represent an updated version of object files (Kahneman, Treisman, \& Gibbs, 1992) to explain how features are bound to objects and enriched by object-related knowledge from long-term memory. In addition to object features, Hommel (2004) showed that the event file can also include codes that apply to action and sensorimotor planning, and in a later study (Hommel, 2005), suggested that features of the stimuli and response are integrated whenever the dimensions that they are defined on are related to the task.

Spence (2009), in summarizing the evidence for the Colavita dominance effect, pointed out that it tends to be associated with tasks that require participants to detect or discriminate the source modality. If this is the case, then perhaps the Colavita dominance effect represents a finding that is limited to instances when visual and auditory events compete for the same processing capacity. It may represent an example of the biased-competition model of selective attention (Desimone \& Duncan, 1995: Duncan, Humphreys, \& Ward, 1997), which predicts that the input that is the most salient would be the one that is analyzed. In this example, the salient bottom-up attribute would be the visual source modality.

To investigate whether visual dominance is restricted only to bimodal conditions in which the audiovisual events compete, we asked participants to detect a target concept that was embedded in a series of rapidly presented stimuli. The target concept could appear as a picture, a sound, or both, and the target frequency replicated the original investigation of visual dominance (Colavita, 1974), with equal unimodal presentations (40\% visual, $40 \%$ auditory) and a smaller number of bimodal events $(20 \%)$. The target and distractor stimuli for both modalities were drawn from subcategory members of living things, tools/machines, and musical instruments. In the unimodal trials, the stimuli appeared in only one format (either as a picture or a sound), and the participants were asked to detect the target with a single keypress. Detection latencies to the visual and auditory targets when they were presented alone would be compared to those from bimodal trials. In the bimodal trials, auditory and visual stimuli were presented simultaneously in one of three conditions. In the consistent condition, the paired audiovisual events (both targets and distractors) were semantically consistent, such as the sound of a guitar playing appearing with the picture of a guitar. In the two inconsistent conditions, however, the audiovisual pairs were drawn from different semantic concepts, and when a 
target appeared, it was paired with a distractor from the other modality. So, if a cat was the target, the picture of a cat could appear together with the sound of a cello (inconsistent sound), or the sound of the cat "meowing" could appear with a picture of a cello (inconsistent picture). By using the same stimulus set for the audiovisual stream and varying only the format of the target and distractor items, it was possible to study crossmodal facilitation and interference effects with fewer confounding factors than in previous work (Sinnett et al., 2008). Yuval-Greenberg and Deouell (2009) also used audiovisual stimuli from the same semantic category, but they required participants to attend to a particular input modality rather than to a semantic concept.

\section{Experiment 1}

Our stimulus set included several exemplars for each item in the stimulus pool, to be sure that detection responses were based on a target concept and not just on perceptual matching to a specific exemplar. For example, Suied et al. (2009) found bimodal facilitation when an object and sound were semantically congruent, and interference when a sound distractor appeared with a semantically incongruent target object, but they only tested one target and distractor object. There is thus no way of distinguishing whether their findings resulted from a target that was conceptually defined or just based on matching a visual or auditory template to the stimulus. Experiment 1 was conducted to test participant responses to the exemplars used for the target categories and to an equal number of distractor items. We wanted to collect some baseline data that would show how the auditory and visual exemplars for the target and distractor items compared when participants used a numerical scale to rate the degree to which an exemplar represented a target concept.

\section{Method}

Participants The participants were 30 (80\% female) undergraduate students from the University of North Carolina at Charlotte, who were 18 years of age or older $\left(M_{\text {age }}=23\right.$, $S D_{\text {age }}=3.90$ ) and had normal hearing and normal (or corrected-to-normal) vision. In all, $43 \%$ of the sample were white, $27 \%$ African American, and $30 \%$ other. They participated to obtain extra credit points toward their psychology class grade. The data from three students were not included in the analysis of the results because of confusion with the response scale and incomplete responses.

Apparatus and materials The stimulus items consisted of pictures and digitized sounds that were selected from databases and clip-art files to represent common and easily recognizable environmental sounds and pictures from the following superordinate categories: musical instruments, tools/machines, or living things.

A list of the items from each category is presented in the Appendix. From these 51 items, three were used as targets (cat, gun, and guitar), and the rest were distractors. The stimulus pool consisted of five exemplars (five different pictures and five different sounds) for each of the target items, and two exemplars for each of the distractors (two different pictures and two different sounds), for a total pool of 111 pictures and 111 sounds. Figure 1 presents the exemplars for each of the picture targets, and the exemplars for the sound targets can be requested from the authors.

Sound files were 32-bit stereo WAV files (sampling rate: $22050 \mathrm{~Hz}$ ) taken from Marcell, Borella, Greene, Kerr, and Roger's (2000) list of 120, and also from the Internet (www. freesound.org). The sounds were edited in Audacity 1.2.5 to a length of $500 \mathrm{~ms}$, and their average amplitude equated to $65 \mathrm{~dB}$ SPL. The pictures were .jpeg files resized using Adobe Photoshop to $4 \times 4 \mathrm{~cm}$. They were selected from clip art, the Internet, and normed lists (Bonin, Peereman, Malardier, Méot, \& Chalard, 2003; Rossion \& Pourtois, 2004). Three observers from the same pool as the participants pretested all of the sound and picture files to ensure $100 \%$ item recognition, and any sounds or pictures that were deemed by the observers to be difficult to discriminate from the targets were removed from the stimulus pool. For example, hammers and jackhammers were removed for confusion with the guns, banjos for confusion with the guitars, and lions for confusion with the cats.

Procedure The participants used a 5-point numerical scale (on which $1=$ not at all and $5=a$ lot) to indicate the degree to which one of 60 stimulus events represented a target concept (either a cat, a gun, or a guitar). Each block of trials consisted of a random arrangement of the ten audio and visual exemplars for one of the three target concepts, together with an equal number of distractor items selected from the same superordinate category (musical instruments, tools/machines, or living things). An instruction screen that identified the target concept with a printed word and the numerical response scale preceded each block of trials. The response scale was visible at the bottom of the monitor at all times during the experiment. The three blocks of trials testing each of the target concepts were presented in the same sequence across participants - cat, gun, and guitar-but the trial sequence within each of the blocks was randomized for each participant.

Each of the 20 trials within a block began with a 500-ms fixation cross that was followed by either an auditory or a visual event. The participants used a numerical keypad to indicate their ratings, and their response terminated the stimulus event. The stimuli were presented on an iMac computer with a 20-in. flat screen. Stimulus presentation and data 
Fig. 1 Exemplars for each of the target pictures. Exemplars of the target sounds are available as supplementary materials, or via e-mail to pagoolka@uncc.edu

\section{CAT}
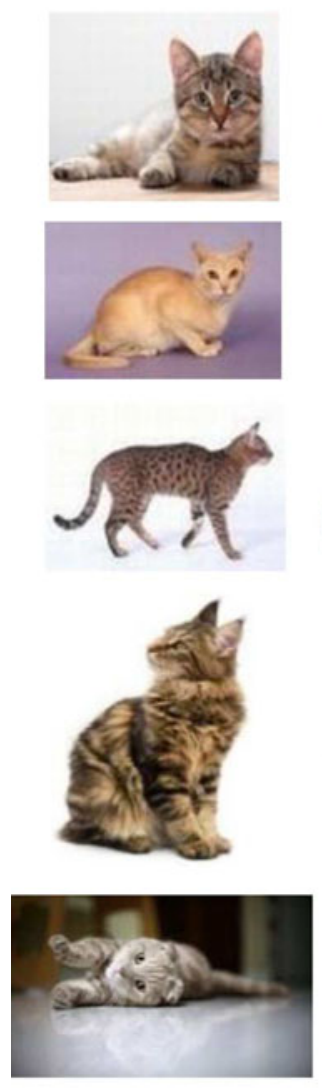

GUITAR
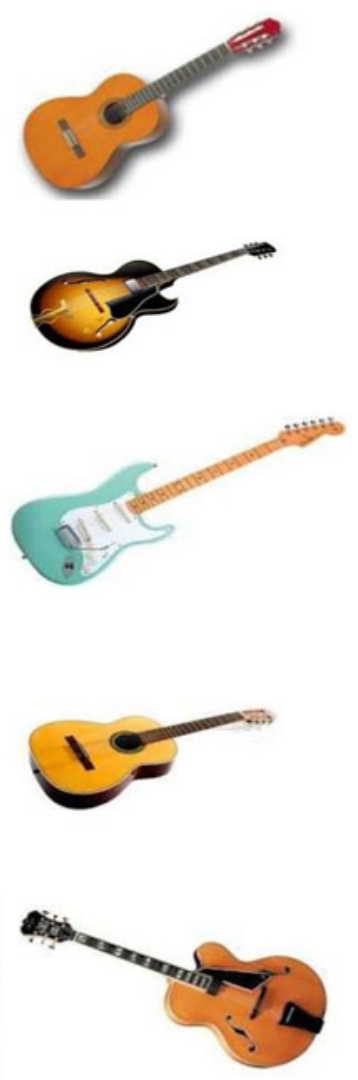

GUN
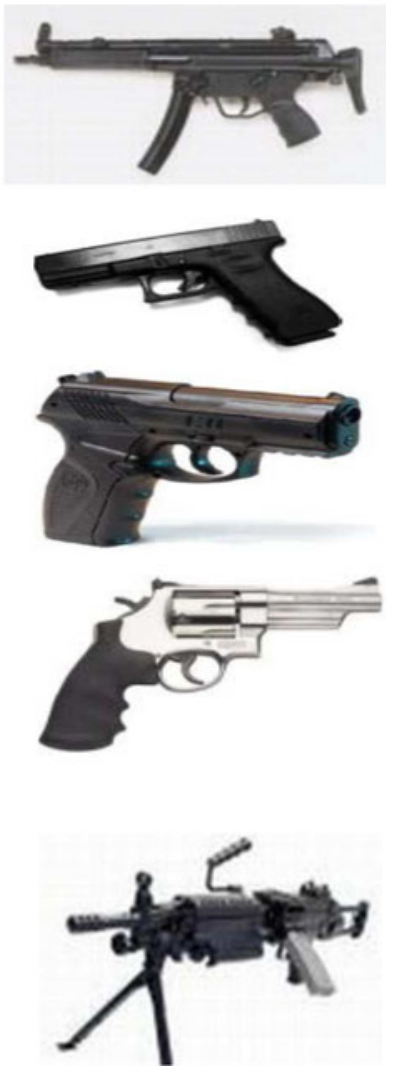

collection were controlled by SuperLab 4.5. The numerical responses and reaction times (RTs) to each of the 60 trials were automatically recorded in a data file.

\section{Results}

The numerical responses and RTs to each of the exemplars of the target and distractor items were averaged across the 27 participants. As expected, those averaged responses indicated that participants, with few exceptions, rated the exemplars of the three target concepts with a 4 or 5 , whereas the exemplars of the distractor items received ratings of 1 or 2 . Table 1 presents the data averaged across the five exemplars in each of the item categories. RTs were trimmed if they were greater than $5,500 \mathrm{~ms}$ (a value greater than two standard deviations $[S D \mathrm{~s}]$ above the mean). Forty-seven $(2.6 \%)$ of the responses were eliminated for this reason.

Separate $2 \times 2$ repeated measures analyses of variance (ANOVAs) were conducted on the numerical responses, and the RTs were averaged across participants and items to identify effects from the audio or visual format of the exemplars and the target/distractor condition. In addition to the obvious
Table 1 Average numerical responses and reaction times (RTs, in milliseconds) to the audiovisual exemplars for each of the target concepts used in Experiment 1

\begin{tabular}{cllll}
\hline Condition & $M_{\text {response }}$ & $S D_{\text {response }}$ & $M_{\mathrm{RT}}$ & $S D_{\mathrm{RT}}$ \\
\hline Distractor & Sounds & & & \\
Cat & 1.26 & 0.79 & 1,760 & 1,004 \\
Guitar & 1.38 & 0.92 & 1,606 & 848 \\
Gun & 1.25 & 0.79 & 1,599 & 853 \\
Pictures & & & & \\
Cat & 1.07 & 0.31 & 1,676 & 1,193 \\
Guitar & 1.39 & 0.68 & 1,481 & 881 \\
Gun & 1.19 & 0.50 & 1,385 & 651 \\
Target & Sounds & & & \\
Cat & 4.83 & 0.54 & 1,700 & 1,123 \\
Guitar & 4.53 & 1.12 & 1,577 & 779 \\
Gun & 4.68 & 0.56 & 1,451 & 892 \\
Pictures & & & & \\
Cat & 4.82 & 0.60 & 1,527 & 1,100 \\
Guitar & 4.90 & 0.26 & 1,084 & 612 \\
Gun & 4.94 & 0.27 & 1,126 & 735 \\
\hline
\end{tabular}

The numerical response scale was from 1 to 5 , in which $1=$ not at all and $5=$ a lot. $n=25$ participants 
effect of condition, $F(1,26)=3,002.72, p<.001, \eta_{\mathrm{p}}{ }^{2}=.99$, the analysis on the numerical responses showed an interaction, $F(1,26)=12.01, p=.002, \eta_{\mathrm{p}}{ }^{2}=.32$, but no main effect of format, $F(1,26)=3.44, p=.075, \eta_{\mathrm{p}}{ }^{2}=.12$. The interaction was due to the higher numerical ratings in response to pictures $(M=4.88)$ than to sounds $(M=4.67)$, but only in the target condition, $t(25)=3.39, p=.002$. We found no format difference in numerical ratings to the distractor items, $t(25)=1.52, p=.140$.

The RT analysis showed significant main effects of condition, $F(1,26)=6.78, p=.015, \eta_{\mathrm{p}}{ }^{2}=.21$, and format, $F(1,26)=$ $21.74, p=.001, \eta_{\mathrm{p}}{ }^{2}=.46$. However, no interaction effect was apparent, $F(1,26)=3.75, p=.086, \eta_{\mathrm{p}}{ }^{2}=.11$ : Picture targets $(M=1,397 \mathrm{~ms})$ were rated more quickly than sounds $(M=1,621 \mathrm{~ms})$, irrespective of whether target or distractor items were presented.

\section{Discussion}

Participant ratings showed that each of the target exemplars consistently represented their respective concepts and that each of the distractor items was similarly rejected as being representative of a concept within that same superordinate category. Also, although both picture and sound stimuli were highly rated by participants as representing a particular semantic concept identified by a printed word, picture targets received slightly higher ratings and were responded to more quickly than sound targets. Although format effects were not evident in the numerical ratings of the distractor items, we did observe format differences in the RTs. These format differences are consistent with past research in our laboratory, with the research of others who found environmental sounds to be more difficult to identify than other stimulus formats (Saygin, Dick, \& Bates, 2005), and with the research of Chen and Spence (2011). They suggested that 200-350 ms are needed for an auditory stimulus to access its semantic representations, and this time is relatively longer than the time needed when pictures are used as the stimuli (Potter, 1975; Thorpe, Fize, \& Marlot, 1996).

\section{Experiment 2}

To investigate the effect of semantic congruity, participants were ask to detect a target concept that was embedded is a series of rapidly presented audiovisual stimuli. Unimodal and bimodal events were randomly mixed in each of the streams. On the basis of the previous findings of a redundant-target effect (Molholm et al., 2004; Sinnett et al., 2008), we expected to find evidence of cross-modal facilitation when the bimodal consistent condition was compared to either of the unimodal conditions: Detection latencies should be shorter when the target was represented in two rather than in one of the modalities. Only in the bimodal inconsistent conditions did we expect to find evidence of interference effects. In particular, a visual dominance effect would be indicated by sound targets that were missed significantly more often when presented together with a visual distractor than in all other conditions. We did not expect, however, that sound distractors would interfere with picture detection. If there is a selective attention bias in favor of visual inputs, then visual distractors would interfere with auditory target detection, but auditory distractors would not produce any interference with picture detection. Given the results of Experiment 1, however, we knew that picture targets would be detected more quickly than environmental sound targets, so we were not interested in overall RT differences between the stimulus formats. Rather, our focus was directed at the proportions of missed targets in the bimodal conditions. The fact that it took participants on average about $311 \mathrm{~ms}$ longer to detect an auditory than a visual target should not prevent environmental sound targets from being detected.

\section{Method}

The participants were 35 (74\% female) students volunteers from the same participant pool as in Experiment 1; they were 18 years of age or older $\left(M_{\mathrm{age}}=24, S D=7.03\right)$ and had normal hearing and normal (or corrected-to-normal) vision. In total, $71 \%$ of the sample were white, $23 \%$ African American, and $6 \%$ other.

Materials and procedure The stimuli were the same as in Experiment 1. Auditory stimuli were delivered through a pair of Labtech Elite 825 stereo headphones. The audiovisual stimuli were presented simultaneously in two continuous

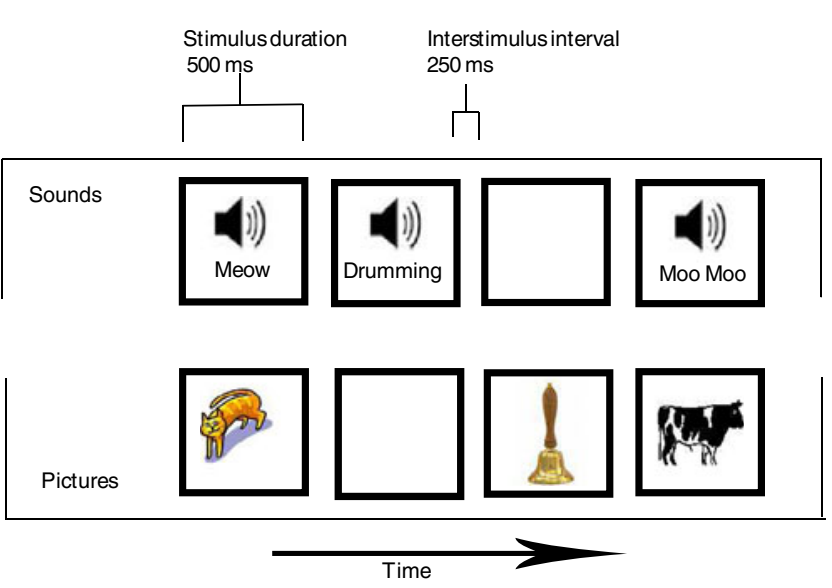

Fig. 2 Sample stream of stimulus items from the bimodal consistent condition. The target item (cat) is presented in both picture and sound formats. Bimodal and unimodal conditions were randomly arranged within each of the streams 
streams, as is indicated in Fig. 2. The unimodal and bimodal conditions were randomly mixed within the stimulus streams. The streams consisted of stimulus durations of $500 \mathrm{~ms}$ separated by interstimulus intervals of $250 \mathrm{~ms}$. Bimodal events included audiovisual pairs of stimuli, whereas unimodal events consisted of a picture or a sound presented with either silence or a blank screen in the other modality.

Participants sat $30 \mathrm{~cm}$ from the computer screen in a welllighted room, wore stereo headphones, and were run individually in 30-min sessions. Prior to the experimental trials, the exemplars for each of the three target concepts appeared one after the other for $500 \mathrm{~ms}$, to familiarize participants with each sound and picture target. For each stream of trials only one target concept was used, and that target concept was designated with the printed word placed on an instruction screen that began each stream of trials. Participants were told to observe the continuous audiovisual stream and to use a single keypress (F) to detect the predefined target as quickly and accurately as possible. Figure 2 shows the timing of each event within a trial.

Each of the audiovisual streams contained 100 trials, and the makeup of those trials replicated the presentation parameters used in the original demonstration of visual dominance (Colavita, 1974) - $40 \%$ unimodal auditory, $40 \%$ unimodal visual, and $20 \%$ bimodal. The targets were embedded in the stimulus stream in random locations approximately every fourth stimulus. In each stream, ten auditory-only targets, ten visual-only, and five bimodal targets were presented. The remaining trials in the stream were distractor trials $(30$ auditory-only, 30 visual-only, and 15 bimodal). The 100 trials were arranged in a random sequence that changed each time the stream was presented.

Three kinds of audiovisual streams were tested, and in each the makeup of the trials was the same, with the exception of the 20 bimodal trials. In the streams that included a bimodal consistent condition, the five target and 15 distractor trials presented the same concept in both modalities concurrently. For example, the picture of a cat would have been paired with the sound of a cat meowing. Similarly, in a distractor trial, the picture of a boat could be presented with the sound of a boat horn. The other two kinds of streams included the bimodal inconsistent conditions, which involved an audiovisual pairing that represented different concepts. In the bimodal inconsistent visual condition, the target appeared as a sound and was paired with a picture from a distractor concept. Similarly, in the bimodal inconsistent auditory condition, the target appeared as a picture and was paired with a distractor sound. For example, the target sound of a cat could be paired with the distractor picture of a piano, or the distractor sound of a piano with the target picture of a cat. For the distractor trials in the bimodal inconsistent conditions, the audiovisual pair presented simultaneously was drawn from different distractor concepts.
The three kinds of audiovisual streams were each presented three times, for a total of nine stimulus streams in the experimental session. The order of the streams was random, with the constraint that each kind occurred once each at the beginning, middle, and end of the experimental session. Each of the three target concepts (cat, gun, and guitar) was used once in each type of stream.

Across all nine stimulus streams, 90 audio-only and 90 visual-only target trials were presented, as well as 15 trials in each of the three bimodal conditions. RTs were measured from the onset of the target stimulus until the participant's keypress response. RTs were counted as target responses if they occurred within 150-1,250 ms after the onset of the target stimulus. Mean RTs were calculated for each participant in every condition. Also noted were the number of misses in each of the conditions (the number of targets that were not detected) and the false alarms (incorrect detection responses to the distractor items).

\section{Results}

RTs and proportions of misses were averaged across the participants and analyzed with separate repeated measures ANOVAs to test for differences among the five bimodal and unimodal conditions. The $F$ tests that are reported include the Greenhouse-Geisser correction to the $p$ value when necessary to protect against possible violation of the sphericity assumption. When significant $F$ values were obtained, post-hoc Bonferroni tests followed up to locate differences among the conditions. A significance level of .05 was used for all statistical tests.

The average proportions of false alarms to the distractor items were low (less than .05) in each of the experimental conditions. However, a repeated measures ANOVA found that these means differed, $F(2,68)=18.49, p<.001, \eta_{\mathrm{p}}{ }^{2}=.35$. Bonferroni tests showed that false alarms were significantly higher when bimodal distractors were used $(M=.04$, $S D=.03)$, in comparison to either visual $(M=.03$, $S D=.02)$ or sound $(M=.02, S D=.02)$ distractors.

Reaction time The upper panel of Fig. 3 presents the mean RTs for detecting the targets across each of the five bimodal and unimodal target conditions. The ANOVA revealed significant differences among the conditions, $F(4,136)=83.28, p<$ $.0001, \eta_{\mathrm{p}}{ }^{2}=.71$. Post-hoc Bonferroni tests showed that the RTs to the bimodal consistent target trials were significantly faster $(M=438 \mathrm{~ms}, S D=35.22)$ than those of both unimodal picture $(M=468 \mathrm{~ms}, S D=32.17)$ and unimodal auditory $(M=$ $528 \mathrm{~ms}, S D=42.14$ ) target trials, and also faster than the other two bimodal conditions, with the inconsistent distractors (i.e., pictures presented with sound distractors $[M=475 \mathrm{~ms}, S D=$ $34.00]$ and sounds presented with picture distractors $[M=$ $533 \mathrm{~ms}, S D=58.63])$. Picture targets were responded to more 
Fig. 3 Mean reaction times and proportions of missed targets in each of the conditions in Experiment 2. Vertical bars represent $95 \%$ confidence intervals
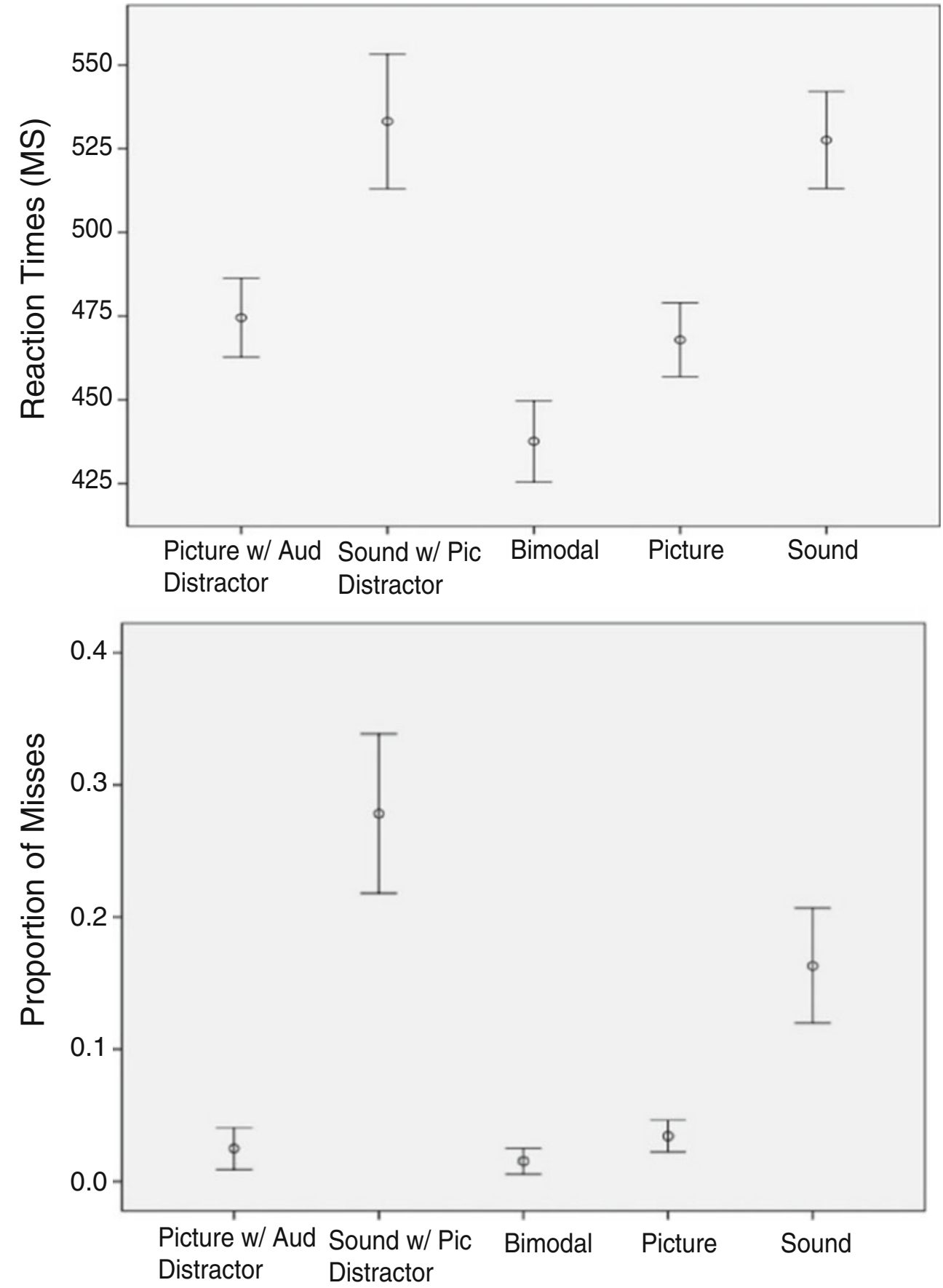

quickly than sound targets, whether the target was presented alone or with an inconsistent distractor. Also, RTs were not affected by the presence of distractors when detecting either the picture or the sound targets.

Misses The lower panel of Fig. 3 presents the average proportions of misses to the target concepts across each of the conditions. The ANOVA on these data also showed a significant effect of condition, $F(4,136)=62.20, p<.001, \eta_{\mathrm{p}}{ }^{2}=.65$. Follow-up tests showed that auditory targets, when presented with visual distractors, were missed significantly more often
$(M=.28, S D=.17)$ than targets in any other condition. Also, when the auditory targets were presented alone, the proportion of misses $(M=.16, S D=.12)$ was significantly higher than in any of the other conditions that included picture targets.

\section{Discussion}

The two notable findings in these results are the faster detection latencies when the bimodal consistent trials were 
compared to both unimodal conditions, and the higher rate of missed targets when visual distractors were presented together with auditory targets, in comparison to the auditory targets presented alone. The first finding, of a facilitation effect associated with the representation of a target concept in two modalities rather than one, is consistent with the redundant-target effect of sensory facilitation, and it is relevant because it extends the finding to include instances in which target detection involves matching bimodal information to a semantic concept. In contrast to the findings of Koppen et al. (2008), semantic congruency did influence the Colavita dominance effect, and presenting semantically congruent information in both audio and visual formats benefited detection of a target concept.

The second finding showed that evidence for visual dominance was restricted to the bimodal inconsistent trials in which sound targets appeared with visual distractors. Although the presence of the visual distractors did not delay RTs to the sound targets, as indicated by the similarity in RTs when this condition was compared to auditory targets presented alone, the higher rate of misses in this condition in comparison to the others clearly showed that the presence of the visual distractor interfered with detection accuracy. Moreover, the fact that auditory distractors did not interfere with picture target detection indicates an asymmetry in crossmodal influences that can be explained by the biasedcompetition theory of attention (Desimone \& Duncan, 1995; Duncan et al., 1997). Attention is a form of competition among the bimodal inputs, and when the inputs are incongruent, the one that gets processed is the one that is the most salient. Yuval-Greenberg and Deouell (2009) found a similar asymmetry in cross-modal influences when they required attention to a specific input modality, and they showed, moreover, that when the visual stimuli were low contrast, and thus became less salient, that the interference effect was eliminated.

The implication of these findings is a tendency to favor visual over auditory inputs only when some incongruity is present in the audiovisual event and the visual component is more salient. The findings from Experiments 1 and 2 are consistent in showing that the basis for the increased saliency associated with the picture rather than the audio format may be that pictures are rated as being more representative of a semantic concept than are sounds, and when searching for a conceptual target, visual representations are responded to more readily than auditory representations, whether they are presented alone or with distractors presented in another modality.

As a further test of the biased-competition model of selective attention as an explanation of our findings in Experiment 2, we wanted to measure whether the evidence for visual dominance would be diminished when we reduced the saliency of the visual stimuli, as had been done in the research of YuvalGreenberg and Deouell (2009). The most limiting factor of the findings from Experiment 2 was the fact that the pictures were rated as being significantly more representative of the semantic concept than were the environmental sounds. Although we expected to find RT differences, on the basis of past findings that compared pictures to environmental sounds, we did not necessarily expect that the numerical ratings of the representativeness of the semantic concept would differ. In an effort to equate the picture and sound representations, we added uniform noise to the pictures to make them slightly less discriminable than the ones used in Experiment 2. The following two experiments were conducted with these filtered picture stimuli in order to assess their effects on participant ratings (Exp. 3) and on the visual dominance effect (Exp. 4).

\section{Experiment 3}

Experiment 3 was conducted to measure participant responses to the filtered visual exemplars for the target and distractor items, and to compare them to responses to the auditory exemplars used in the previous experiments. Participant ratings would be used to determine whether the addition of a noise filter to the pictures would take away the slight advantage that pictures had in comparison to auditory exemplars as being representative of a target concept.

\section{Method}

A group of 26 (73\% female) volunteers from the same participant pool were used. They were 18 years of age or older $\left(M_{\text {age }}=22, S D=5.80\right)$, with normal (or corrected-to-normal) vision and hearing. In all, $38 \%$ of the sample were white, $46 \%$ African American, and $16 \%$ other. The students had not participated in the previous experiments, and the data from one were not included in the analysis because of failure to complete the experiment.

The stimulus items were the pictures and digitized sounds that had been used in Experiment 1; however, the pictures were altered in Adobe Photoshop by adding a uniform noise filter at $50 \%$ strength to each of the jpeg files. Figure 4 shows some of the target pictures that had been changed in this manner. In all other respects, this experiment was the same as Experiment 1.

Participants used a 5-point numerical scale to indicate the degree to which one of 60 stimulus events represented a target concept (either a cat, a gun, or a guitar). Each block of trials consisted of a random arrangement of the ten audio and visual exemplars for one of the three target concepts, together with an equal number of distractor items.

\section{Results and discussion}

Numerical responses and RTs to each of the exemplars of the target and distractor items were averaged across the 25 
Fig. 4 Sample target stimuli from Experiments 3 and 4, with $50 \%$ noise added

participants. Consistent with the results of Experiment 1, those averaged responses indicated that participants rated the exemplars of the three target concepts with a 4 or 5 , whereas the exemplars of the distractor items received ratings of 1 or 2 , for the most part. Table 2 presents the data averaged across the five exemplars in each of the item categories. As before, RTs

Table 2 Average numerical responses and reaction times (RTs, in milliseconds) to the audiovisual exemplars for each of the target concepts used in Experiment 3

\begin{tabular}{lllll}
\hline Condition & $M_{\text {response }}$ & $S D_{\text {response }}$ & $M_{\mathrm{RT}}$ & $S D_{\mathrm{RT}}$ \\
\hline $\begin{array}{c}\text { Distractor } \\
\text { Cat }\end{array}$ & 1.27 & 0.77 & Sounds & \\
Guitar & 1.34 & 0.66 & 1,949 & 928 \\
Gun & 1.20 & 0.55 & 1,806 & 752 \\
& & & Pictures & \\
Cat & 1.17 & 0.61 & 2,170 & 1,227 \\
Guitar & 1.61 & 0.75 & 1,841 & 1,181 \\
Gun & 1.27 & 0.52 & 1,738 & 885 \\
Target & & & Sounds & \\
Cat & 4.66 & 0.83 & 1,976 & 1,063 \\
Guitar & 4.58 & 0.95 & 1,836 & 831 \\
Gun & 4.58 & 0.95 & 1,720 & 909 \\
& & & & \\
Cat & 4.62 & 0.94 & 968 & 967 \\
Guitar & 4.92 & 0.30 & 1,221 & 698 \\
Gun & 4.60 & 0.66 & 1,535 & 1,039 \\
\hline
\end{tabular}

The numerical response scale is from 1 to 5 , in which $1=$ not at all and $5=$ a lot. $n=25$ participants were trimmed if they were greater than $5,500 \mathrm{~ms}$ (a value greater than $2 S D$ s above the mean). Thirty-five $(2.3 \%)$ of the responses were eliminated for this reason.

The ANOVA on the numerical responses showed only a main effect of target/distractor condition, $F(1,24)=1,783.47$, $p<.001, \eta_{\mathrm{p}}{ }^{2}=.99$. We found neither a main effect of format, $F(1,24)=2.71, p=.11, \eta_{\mathrm{p}}{ }^{2}=.10$, nor an interaction, $F<1$. The addition of the noise filter to the pictures was successful in reducing the picture advantage in the numerical ratings that we had observed in Experiment 1: Our participants rated the filtered picture and sound target stimuli as being similarly representative of the target concept.

The results from the RTs analysis, however, were consistent with those of Experiment 1. RTs were faster when pictures ( $M=1,690 \mathrm{~ms})$ rather than sounds $(M=1,882 \mathrm{~ms})$ were rated, $F(1,24)=4.83, p<.038, \eta_{\mathrm{p}}{ }^{2}=.17$, and for targets rather than distractors, $F(1,24)=5.50, p<.028, \eta_{\mathrm{p}}{ }^{2}=.19$. The interaction between these variables did not reach significance, $F(1,24)=$ 2.07, $p=.16, \eta_{\mathrm{p}}{ }^{2}=.08$.

\section{Experiment 4}

Since we were successful in Experiment 3 in showing that the filtered visual exemplars and environmental sounds were rated to be similarly representative of the semantic concepts under study, we repeated Experiment 2 with these stimuli. Following the example of Yuval-Greenberg and Deouell (2009), we wanted to test whether a reduction in the saliency of the visual stimuli would reduce the interference caused by the visual distractors on audio target detection. 
A second change that was made in order to equalize the impacts of the visual and audio stimuli was to extend the interstimulus interval from $250 \mathrm{~ms}$ (used in Exp. 2) to $500 \mathrm{~ms}$. This was done to provide more time to process each of the target events. Given the relatively longer time needed to access semantic information when sounds are used, as compared to pictures (Chen \& Spence, 2011), we wanted to make sure that the modality differences in the findings from Experiment 2 were not due to insufficient time being available for the auditory events to be processed.

As in the previous Experiment 2, participants were asked to detect a target concept that was embedded in a series of rapidly presented audiovisual stimuli. We were interested in whether the evidence from Experiment 2 for the Colavita dominance effect would still be present even though the picture were rated as being similar to the auditory stimuli in semantic representativeness, and even when more time to process the target events was provided. Biased-competition theory would suggest that our efforts at equalizing the influences of the audio and visual stimuli should reduce the saliency of the pictures and, as a result, their capacity to interfere with processing of the sound targets when audio and visual events were presented simultaneously. Supporting evidence would be found if the higher rate of missed targets in the condition when visual distractors appeared together with audio targets (in comparison to the unimodal sound target condition) was reduced or eliminated.

\section{Method}

A group of 31 ( $71 \%$ female) volunteers were drawn from the same participant pool as in the previous experiments. The students were 18 years of age or older $\left(M_{\mathrm{age}}=20\right.$, $S D=2.49$ ) and had normal (or corrected-to-normal) vision and hearing. In total, $52 \%$ of the sample were white, $29 \%$ African American, and $19 \%$ other. None of the students had participated in any of the previous experiments.

The stimuli were the 111 sounds used in the previous experiments and the 111 filtered pictures from Experiment 3. The audiovisual stimuli were presented simultaneously in two continuous streams, with randomly mixed unimodal and bimodal conditions. As in Experiment 2, the stream consisted of stimulus durations of $500 \mathrm{~ms}$; however, the interstimulus interval was lengthened to $500 \mathrm{~ms}$. In all other respects, this experiment was run in the same manner as Experiment 2.

\section{Results and discussion}

Consistent with the previous experiment, false alarms to the distractor items were infrequent, but the mean proportions varied significantly by distractor type, $F(2,60)=9.93$, $p<.01, \eta_{\mathrm{p}}{ }^{2}=.25$. Follow-up tests showed that false alarms to the auditory distractors $(M=.01, S D=.01)$ were significantly lower than false alarms in response to either bimodal $(M=.02, S D=.02)$ or visual $(M=.02, S D=.01)$ distractors.

Reaction time Fig. 5 presents the mean RTs to detecting the target in each of the conditions. As in the previous experiment, RTs were measured from the onset of the target stimulus until the participant's keypress response and were counted as target responses if they occurred within $150-1,250 \mathrm{~ms}$ after the onset of the target stimulus.

RTs varied significantly among the conditions, $F(4,120)=$ 54.63, $p<.001, \eta_{\mathrm{p}}{ }^{2}=.65$, and post-hoc Bonferroni tests showed that responses to bimodal targets were significantly faster $(M=466 \mathrm{~ms}, S D=38.41)$ than those in the other conditions. RTs were faster to the picture targets than to the sound targets, whether the pictures were presented alone ( $M=$ $502 \mathrm{~ms}, S D=31.75)$ or with a sound distractor $(M=497 \mathrm{~ms}$, $S D=39.70$ ); also, we found no RT differences to the sound targets, whether they were presented alone $(M=553 \mathrm{~ms}, S D=$ $55.18)$ or with a picture distractor $(M=571 \mathrm{~ms}, S D=76.93)$.

The RT results essentially replicated the findings from Experiment 2: Audiovisual targets were responded to more quickly than those in either of the unimodal target conditions, and pictures targets yielded quicker responses than auditory targets. Target processing in both of the modalities tested was not delayed by the presence of inconsistent distractors presented in a different modality.

Misses The bottom panel of Fig. 5 presents the average proportions of misses to the target concepts across each of the conditions. Consistent with Experiment 2, we observed considerable variability in the proportions of misses across the unimodal and bimodal conditions, $F(4,120)=17.60, p<.001$, $\eta_{\mathrm{p}}{ }^{2}=.37$. Follow-up tests showed significant differences ( $p=.05$ significance level) between the proportions of misses in the bimodal target condition and in all others, and between the conditions with picture targets and sound targets. We found a low incidence of misses with bimodal targets $(M=.01, S D=.02)$, and misses were only slightly more prevalent when picture targets appeared alone $(M=.04$, $S D=.05)$ or with sound distractors $(M=.05, S D=.07)$. Importantly, however, sound targets presented with picture distractors $(M=.15, S D=.15)$ were missed about as often as when the sound targets appeared alone $(M=.11, S D=.10)$. No significant differences emerged in the proportions of misses when either the audio or the visual targets were presented alone or together with distractors from the other modality.

These data differ importantly from the findings in Experiment 2, due to the fact that the proportion of misses did not increase significantly when the audio targets were presented together with visual distractors. The addition of the visual noise filter to the pictures, together with the extension of the interstimulus interval duration, may have made the 
Fig. 5 Mean reaction times and proportions of misses in each of the target conditions in Experiment 4. Vertical bars represent $95 \%$ confidence intervals
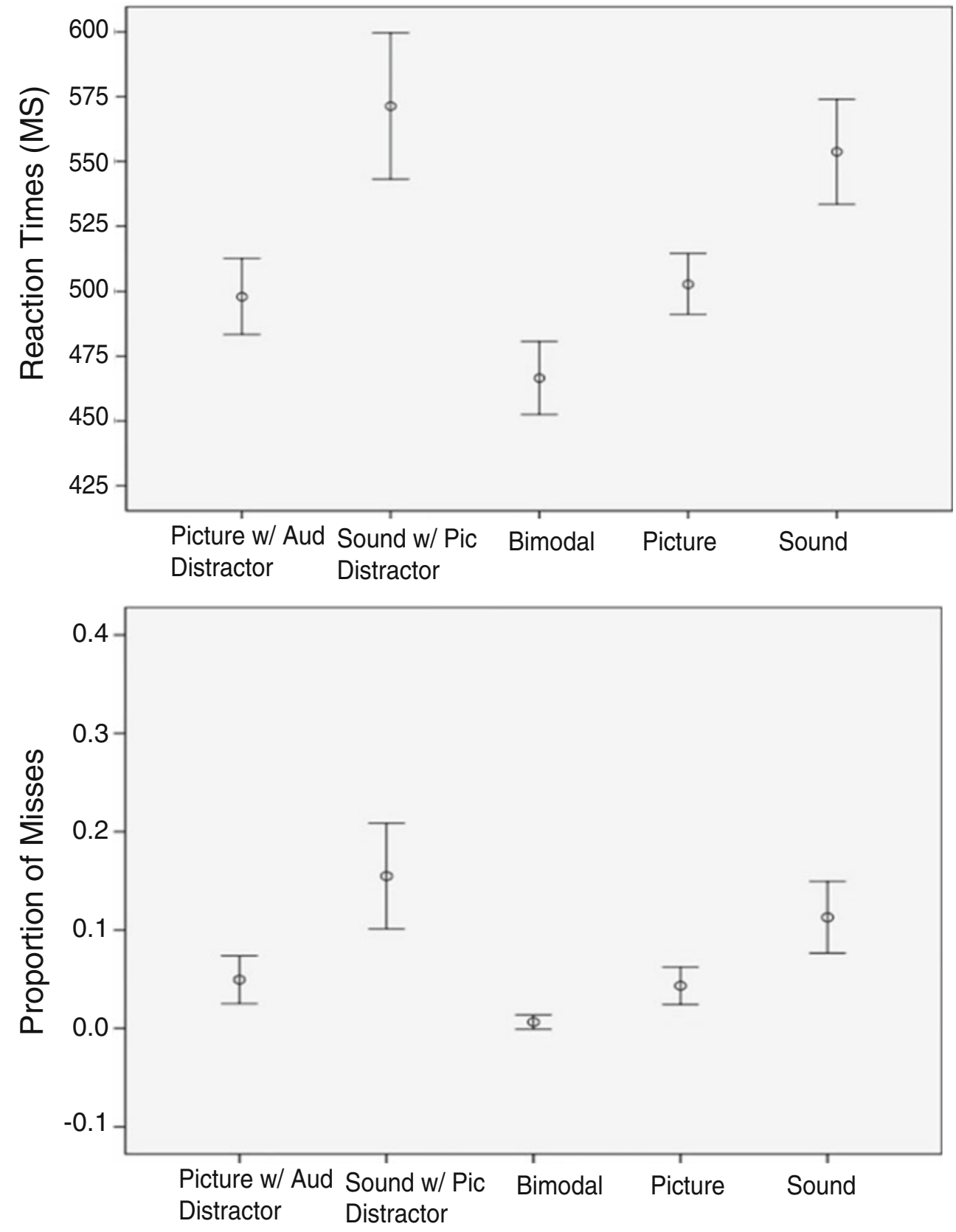

sound and picture stimuli more equivalent in terms of saliency than they had been in Experiment 2, and as a result, the evidence for visual dominance was diminished.

\section{General discussion}

Taken together, the findings from Experiments 2 and 4 are consistent in showing a facilitation effect associated with the representation of a target concept in two modalities rather than one. The faster detection latencies that were obtained in the bimodal consistent trials, when compared to both unimodal conditions, replicate the redundant-target effect of sensory facilitation (Molholm et al., 2004; Sinnett, et al., 2008) and extend the effect to the detection of a target concept. Presenting semantically congruent information in both audio and visual formats benefited detection of a target concept.

The evidence in support of a visual dominance effect, however, that was obtained in Experiment 2 was much diminished in the findings of Experiment 4 . We found no evidence for cross-modal influences on semantic target detection when efforts were made to equalize the impacts of the pictures and environmental sounds by degrading the picture stimuli and 
lengthening the target processing time. This finding is consistent with the predictions of the biased-competition theory of attention and with the results of Yuval-Greenberg and Deouell (2009), who found a reduction in interference, in association with a reduction in saliency of the visual stimuli.

Visual dominance does not appear to be a characteristic of how audiovisual events are processed in general. Taken together, these findings support the event file (Hommel, 2004, 2005) as the mechanism that underlies multimodal sensory integration, because our results show facilitation when bimodal features are congruent and interference when they are incongruent; however, there is no doubt that concept detection is faster and more accurate with visual than with auditory representations.

The fact that visual targets had shorter detection latencies and fewer missed responses than auditory targets, regardless of whether they were presented alone or with a distractor, is at variance with other demonstrations of the Colavita dominance effect (Colavita, 1974) in which the detection RTs for visual and auditory targets were similar. Those studies, however, used a detection task with simple audiovisual stimuli (such as the presence of a tone or light). With more complex identification tasks, like the concept identification task that we used, visual targets are processed more quickly than sounds, because visual information is available all at once whereas auditory stimuli unfold over time, and when participants are asked to process the content of the information, this takes longer with auditory stimuli, as demonstrated by the findings from Experiments 1 and 3, and as had been found by other researchers who compared environmental sounds and pictures (Chen \& Spence, 2011; Saygin et al., 2005).

The results of the present study showed both sensory facilitation and interference (Exp. 2) within the same task, just as in Sinnett et al. (2008). However, these results were found in response to a detection task with a semantic target rather than a task requiring discrimination of format modality, as in Sinnett et al.'s (2008) study. The use of several exemplars of the stimulus pool items ensured that detections were made in response to a semantic concept, rather than just perceptual matching to a specific exemplar item. Also, taking the visual and auditory stimuli from the same stimulus set as opposed to different sets allowed for less confounding between conditions, since the same semantically congruent categories and items were equally represented in all conditions. In the natural world, not all cats look the same, and they are not always in the same position or seen from the same perspective angle. They also do not all sound the same, or have the same tone with every vocalization. By having varying exemplars, the idea of "cat" is better represented, rather than confining the item to being solely defined by a single picture and sound. Therefore, this method increased the external validity of the project in comparison to a previous study (Suied et al., 2009). The results show that facilitation and interference are not only found in a simple detection task, but in dealing with identifying members of a semantically conceptual category, as well. Additionally, this finding extends the results of Ngo et al. (2010) by demonstrating evidence for the Colavita visual dominance effect with a conceptual task, and it is consistent with other studies that have shown the positive influence of semantic congruency when targets are bimodal (Chen \& Spence, 2010; Molholm et al., 2004).

So, in revisiting the Colavita dominance effect, we found supporting evidence, even when we used a task that required access to a semantic concept. However, when efforts were made to equalize the saliency of the auditory and visual stimulus representations of the target concept, then the supporting evidence diminished. We suggest that future researchers consider the relative differences in saliency and RTs when working with multimodal stimulus formats.

These findings are, of course, limited by the nature of the stimulus items that we used - commonly recognized nouns and environmental sounds - and by the fact that only college students were tested. Whether they can be generalized to broader categories of participants and stimulus materials will only be determined by more research.

Author Note Thanks are due Jennifer Feagans and Hilary Haagen for their assistance with this project.

\section{Appendix}

\begin{tabular}{lll}
\hline Animals & Musical Instruments & $\begin{array}{l}\text { Tools/Machines } \\
\text { boat } \\
\text { bird }\end{array}$ \\
cat & bagpipes & car \\
chicken & banjo & cash register \\
cow & bell & gun \\
cricket & bongos & hammer \\
crow & cello & jackhammer \\
dog & clarinet & motorcycle \\
donkey & cymbal & saw \\
duck & drum & scissors \\
elephant & flute & siren \\
frog & gong & stapler \\
hawk & guitar & sword \\
horse & harmonica & \\
monkey & harp & \\
owl & maracas & \\
pig & organ & \\
rooster & piano & \\
seal & saxophone & \\
sheep & trumpet & \\
turkey & violin & \\
woodpecker & wind chime & \\
\hline
\end{tabular}




\section{References}

Bonin, P., Peereman, R., Malardier, N., Méot, A., \& Chalard, M. (2003). A new set of 299 pictures for psycholinguistic studies: French norms for name agreement, image agreement, conceptual familiarity, visual complexity, image variability, age of acquisition, and naming latencies. Behavior Research Methods, Instruments, \& Computers, 35, 158-167. doi:10.3758/BF03195507

Chen, Y., \& Spence, C. (2010). When hearing the bark helps to identify the dog: Semantically-congruent sounds modulate the identification of masked pictures. Cognition, 114, 389-404. doi:10.1016/j. cognition.2009.10.012

Chen, Y., \& Spence, C. (2011). Crossmodal semantic priming by naturalistic sounds and spoken words enhances visual sensitivity. Journal of Experimental Psychology, 37, 1554-1568.

Colavita, F. B. (1974). Human sensory dominance. Perception \& Psychophysics, 16, 409-412.

Desimone, R., \& Duncan, J. (1995). Neural mechanisms of selective visual attention. Annual Review of Neuroscience, 18, 193-222. doi:10.1146/annurev.ne.18.030195.001205

Duncan, J., Humphreys, G., \& Ward, R. (1997). Competitive brain activity in visual attention. Current Opinion in Neurobiology, 7, 255-261.

Hommel, B. (2004). Event files: Feature binding in and across perception and action. Trends in Cognitive Sciences, 8, 494-500. doi:10.1016/j. tics.2004.08.007

Hommel, B. (2005). How much attention does an event file need? Journal of Experimental Psychology: Human Perception and Performance, 31, 1067-1082.

Kahneman, D., Treisman, A., \& Gibbs, B. J. (1992). The reviewing of object files: Object-specific integration of information. Cognitive Psychology, 24, 175-219. doi:10.1016/0010-0285(92)90007-O

Koppen, C., Alsius, A., \& Spence, C. (2008). Semantic congruency and the Colavita visual dominance effect. Experimental Brain Research, 184, 533-546.

Lee, F. H., \& Chan, A. S. (2008). Ergonomics recommendations for simultaneous and delayed presentation of visual and auditory signals. Displays, 29, 124-131. doi:10.1016/j.displa. 2007.09.006

Marcell, M. M., Borella, D., Greene, M., Kerr, E., \& Rogers, S. (2000). Confrontation naming of environmental sounds. Journal of Clinical and Experimental Neuropsychology, 22, 830-864.
Molholm, S., Ritter, W., Javitt, D. C., \& Foxe, J. J. (2004). Multisensory visual-auditory object recognition in humans: A high-density electrical mapping study. Cerebral Cortex, 14, 452-465.

Murray, M. M., \& Spierer, L. (2009). Auditory spatio-temporal brain dynamics and their consequences for multisensory interactions in humans. Hearing Research, 258, 121-133. doi:10.1016/j.heares. 2009.04.022

Ngo, M. K., Sinnett, S., Soto-Faraco, S., \& Spence, C. (2010). Repetition blindness and the Colavita effect. Neuroscience Letters, 480, 186 190. doi:10.1016/j.neulet.2010.06.028

Potter, M. C. (1975). Meaning in visual search. Science, 187, 965-966. doi:10.1126/science. 1145183

Rossion, B., \& Pourtois, G. (2004). Revisiting Snodgrass and Vanderwart's object pictorial set: The role of surface detail in basic-level object recognition. Perception, 33, 217-236. doi:10. 1068/p5117

Saygin, A. P., Dick, F., \& Bates, E. (2005). An on-line task for contrasting auditory processing in the verbal and nonverbal domains and norms for younger and older adults. Behavior Research Methods, 37, 99 110. doi:10.3758/BF03206403

Sinnett, S., Soto-Faraco, S., \& Spence, C. (2008). The co-occurence of multisensory competition and facilitation. Acta Psychologica, 128, 153-161. doi:10.1016/j.actpsy.2007.12.002

Sinnett, S., Spence, C., \& Soto-Faraco, S. (2007). Visual dominance and attention: The Colavita effect revisited. Perception \& Psychophysics, 69, 673-686. doi:10.3758/BF03193770

Spence, C. (2009). Explaining the Colavita visual dominance effect. Progress in Brain Research, 176, 245-258. doi:10.1016/Soo796123(09)17615-X

Suied, C., Bonneel, N., \& Viaud-Delmon, I. (2009). Integration of auditory and visual information in the recognition of realistic objects. Experimental Brain Research, 194, 91-102. doi:10.1007/s00221008-1672-6

Thorpe, S., Fize, D., \& Marlot, C. (1996). Speed of processing in the human visual system. Nature, 381, 520-522. doi:10.1038/381520a0

Yuval-Greenberg, S., \& Deouell, L. Y. (2009). The dog's meow: Asymmetrical interaction in cross-modal object recognition. Experimental Brain Research, 193, 603-614. doi:10.1007/s00221008-1664-6

Zmigrod, S., Spapé, M., \& Hommel, B. (2009). Intermodal event files: Integrating features across vision, audition, taction, and action. Psychological Research, 73, 674-684. 